\title{
Comparison of Frequency- and Time-Domain Autoregulation and Vasoreactivity Indices in a Piglet Model of Hypoxia-Ischemia and Hypothermia
}

\author{
Rathinaswamy B. Govindan ${ }^{a, b}$ Ken M. Brady ${ }^{c}$ An N. Massaro a, b, d Jamie Perin ${ }^{e}$ \\ Jacky M. Jennings ${ }^{e}$ Adre J. DuPlessis ${ }^{a, b}$ Raymond C. Koehler ${ }^{f}$ Jennifer K. Lee ${ }^{f}$ \\ ${ }^{a}$ Fetal Medicine Institute, Children's National Health System, Washington, DC, USA; ${ }^{b}$ The George Washington University \\ School of Medicine, Washington, DC, USA; ' Department of Anesthesiology, Baylor College of Medicine and Texas \\ Children's Hospital, Houston, TX, USA; ${ }^{\mathrm{d}}$ Neonatology, Children's National Health System, Washington, DC, USA; ${ }^{\mathrm{e} C e n t e r}$ \\ for Child and Community Health Research, Department of Pediatrics, Johns Hopkins University School of Medicine,

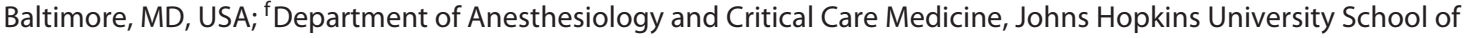 \\ Medicine, Baltimore, MD, USA
}

\section{Keywords}

Brain hypoxia - Cerebrovascular circulation .

Hypothermia $\cdot$ Ischemia $\cdot$ Newborn

\begin{abstract}
Introduction: The optimal method to detect impairments in cerebrovascular pressure autoregulation in neonates with hypoxic-ischemic encephalopathy (HIE) is unclear. Improving autoregulation monitoring methods would significantly advance neonatal neurocritical care. Methods: We tested several mathematical algorithms from the frequency and time domains in a piglet model of HIE, hypothermia, and hypotension. We used laser Doppler flowmetry and induced hypotension to delineate the gold standard lower limit of autoregulation (LLA). Receiver operating characteristics curve analyses were used to determine which indices could
\end{abstract}

distinguish blood pressure above the LLA from that below the LLA in each piglet. Results: Phase calculation in the frequency band with maximum coherence, as well as the correlation between mean arterial pressure (MAP) and near-infrared spectroscopy relative total tissue hemoglobin $(\mathrm{HbT})$ or regional oxygen saturation $\left(\mathrm{rSO}_{2}\right)$, accurately discriminated functional from dysfunctional autoregulation. Neither hypoxia-ischemia nor hypothermia affected the accuracy of these indices. Coherence alone and gain had low diagnostic value relative to phase and correlation. Conclusion: Our findings indicate that phase shift is the most accurate component of autoregulation monitoring in the developing brain, and it can be measured using correlation or by calculating phase when coherence is maximal. Phase and correlation autoregulation indices from MAP and $\mathrm{rSO}_{2}$ and vasoreactivity indices from MAP and $\mathrm{HbT}$ are accurate metrics that are suitable for clinical HIE studies.

\section{KARGER}

E-Mail karger@karger.com

www.karger.com/dne (c) 2019 S. Karger AG, Basel Jennifer K. Lee, MD

Department of Anesthesiology and Critical Care Medicine,

Division of Pediatric Anesthesiology, 1800 Orleans Street, Room 6321

Baltimore, MD 21287 (USA)

E-Mail jklee@jhmi.edu 


\section{Introduction}

Cerebral pressure autoregulation buffers the changes in systemic arterial blood pressure to maintain a steady cerebral blood flow (CBF). Dysfunctional autoregulation with pressure-passive CBF may be a key contributor to secondary brain injury in neonatal hypoxic-ischemic encephalopathy (HIE) [1-5]. Though several techniques can be used to monitor autoregulation, which method produces the greatest accuracy in HIE is unclear. Identifying the most accurate autoregulation monitoring method for use in clinical trials would advance neonatal neurocritical care.

Autoregulation is monitored by detecting the relationship between cerebral perfusion pressure (CPP) and CBF. Autoregulatory vasoreactivity is measured by the relationship between CPP and cerebral blood volume (CBV). In neonates, mean arterial pressure (MAP) is the only accessible clinical indicator of CPP. Continuous measures of $\mathrm{CBF}$ and $\mathrm{CBV}$ in the neonatal clinical setting can be challenging. Transcranial Doppler is commonly used to measure $\mathrm{CBF}$ velocity, and changes in intracranial pressure (ICP) can identify CBV fluctuations [6]. However, Doppler cannot be conducted continuously for multiple days, and continuous ICP monitoring is not possible in most neonates.

Near-infrared spectroscopy (NIRS) can be applied noninvasively and continuously to obtain indicators of $\mathrm{CBF}$ and $\mathrm{CBV}$ that are used to measure autoregulation and vasoreactivity [6-8]. The regional cortical oxygen saturation $\left(\mathrm{rSO}_{2}\right)$ correlates with $\mathrm{CBF}$ during periods of stable arterial oxygen saturation and steady cerebral metabolic rate [9-11]. Fluctuations in relative total tissue hemoglobin (HbT), which is the sum of oxygenated and deoxygenated hemoglobin, can detect regional changes in CBV $[6,11,12]$.

Multiple mathematical approaches are used to interrogate the relationship between blood pressure and CBF or $\mathrm{CBV}$ and generate autoregulation and vasoreactivity indices. These approaches can be classified into frequency $[13,14]$ and time [15] domain analyses. Autoregulation has a characteristic impulse-response time that makes the frequency domain attractive because these methods give frequency-specific results. In time-domain analysis, the signals are filtered in the frequency band of cerebral pressure autoregulation before the correlation between perfusion pressure and CBF or CBV is calculated, providing a similar frequency-specific measure. Though both domains have been used to study autoregulation in HIE [1-4], they are not interchangeable. Direct comparisons between the domains have not been thoroughly tested in HIE. Such a comparison is essential to ensure that the most accurate autoregulation metric is used in clinical research and potentially advanced into clinical practice.

We analyzed data from a piglet model of HIE, hypothermia, rewarming, and controlled hypotension to compare frequency- and time-domain mathematical processing. We used laser Doppler flowmetry (LDF) to measure relative $\mathrm{CBF}$ and calculate each piglet's lower limit of autoregulation (LLA), and thereby distinguish functional from dysfunctional autoregulation $[6,11]$. We hypothesized that the phase calculation in the bandwidth with highest coherence between MAP and $\mathrm{HbT}$ would distinguish blood pressure above the LLA from that below the LLA more accurately than the other indices.

Additionally, hypoxic-ischemic (HI) brain injury and hypothermia affect accuracy in detecting the LLA with NIRS [9-11]. Potential oxidative stress [16] and proinflammatory cytokine shifts [17] during rewarming could affect the magnitude of subsequent vasoreactive responses. If the relative change in vascular responses above the LLA compared to that below the LLA is reduced after rewarming, an index's ability to detect this change and distinguish functional from dysfunctional autoregulation might be reduced. We secondarily tested whether hypoxia-ischemia, hypothermia, and rewarming decrease accuracy in distinguishing blood pressure above or below the LLA relative to that of a sham procedure or normothermia.

\section{Materials and Methods}

We analyzed data from piglets that we have previously reported to conserve animals [9-11]. The Johns Hopkins University Animal Care and Use Committee approved all protocols, and all procedures complied with the United States Public Health Service Policy on the Humane Care and Use of Laboratory Animals and the Guide for the Care and Use of Laboratory Animals. We ensured animal comfort at all points in the protocols.

Within each study [9-11], male piglets (age: 3-5 days; body weight: $1-2.5 \mathrm{~kg}$ ) were randomized to undergo hypoxia-ischemia or a sham procedure (Fig. 1). Some piglets were also randomized to receive normothermia, sustained whole-body hypothermia, or hypothermia and rewarming. Others recovered for 1 or 2 days after hypoxia-ischemia. All piglets with available autoregulation data were analyzed for our study.

\section{Anesthesia and Surgery}

We induced general anesthesia with 5\% isoflurane in 50\%/50\% nitrous oxide/oxygen through a nose cone. The piglets were intubated and mechanically ventilated to maintain normocapnea. A femoral venous catheter and a femoral arterial catheter were placed for intravenous (i.v.) fluids, medications, blood sampling, and 


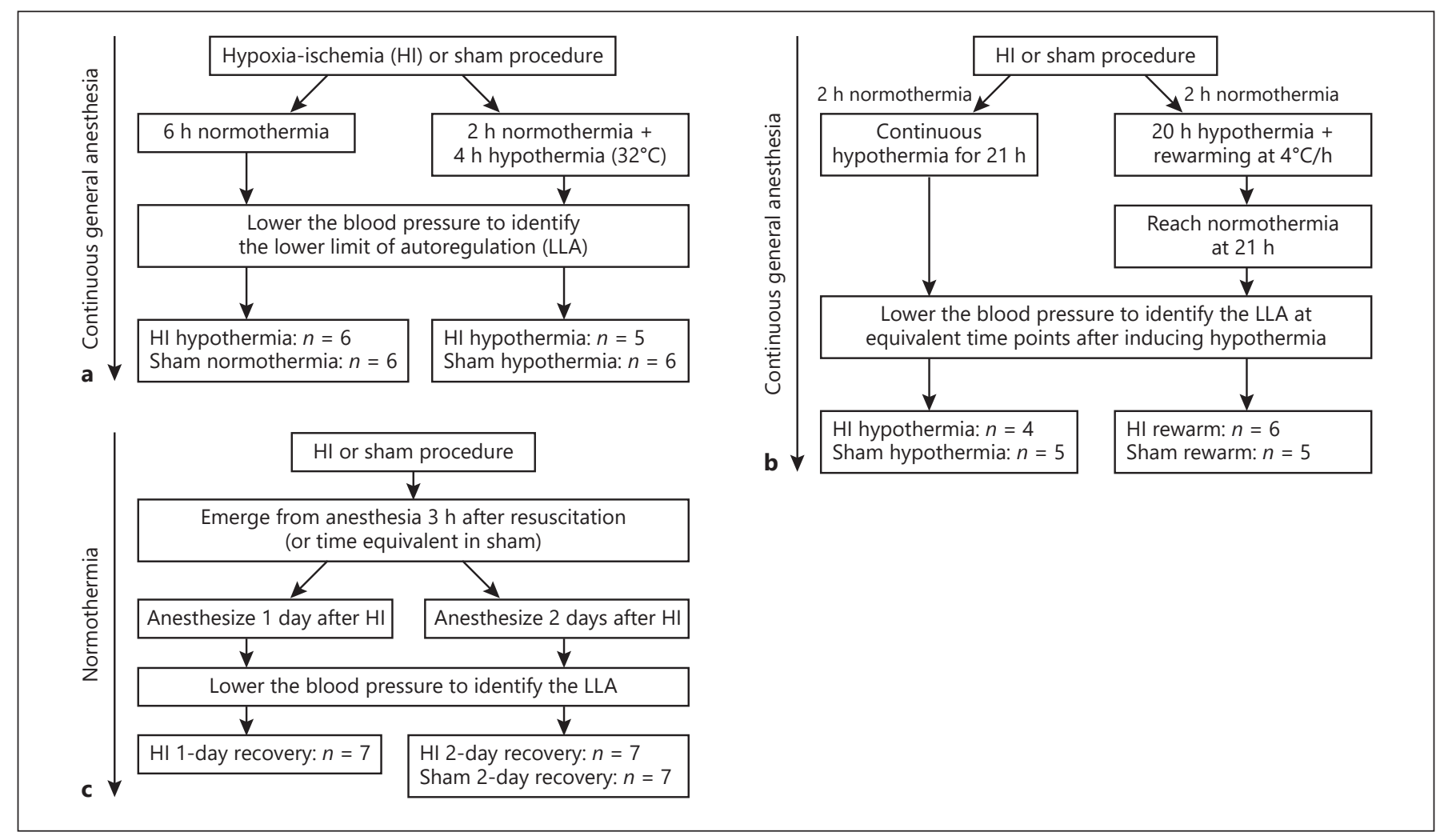

Fig. 1. Data on piglets of 3 previously published studies [9-11] were analyzed. a-c Each study's design and group allocation after randomization.

continuous arterial blood pressure monitoring. Fentanyl $(10 \mu \mathrm{g} / \mathrm{kg}$ bolus $+10-20 \mu \mathrm{g} / \mathrm{kg} / \mathrm{h}$ i.v.) was started, and the isoflurane was decreased to $1.5 \%$. All piglets received vecuronium $(1 \mathrm{mg} / \mathrm{kg} / \mathrm{h})$ to prevent respiratory effort during the hypoxia-asphyxia protocol for hypoxic-ischemic (HI) injury, and to provide the same anesthetic to all groups. A 5-Fr balloon catheter was inserted into the contralateral femoral vein, and then advanced into the inferior vena cava for later inflation to induce controlled hypotension. Three cranial, frontoparietal burr holes ( $\leq 4 \mathrm{~mm}$ each) were made for placement of the laser Doppler probe (Moor Instruments, Devon, UK, model DRT4), ICP monitor, and temperature probe. An NIRS pediatric optode (Covidien, Boulder, CO, USA) was placed on the skin contralateral to the invasive monitors to measure contralateral frontoparietal cortex. We decreased the isoflurane to 0.4 $[10]$ or $0.8 \%[9,11]$ upon completion of surgery. The fentanyl and nitrous oxide were continued for the rest of the experiment.

For the cohort of piglets that emerged from anesthesia, we reinduced anesthesia as described above 1 or 2 days after hypoxiaischemia or the sham procedure (Fig. 1c). The femoral 5-Fr venous balloon catheter and intracranial monitors were placed during the second anesthetic.

\section{HI Brain Injury}

We previously published our HI injury protocol [18]. This protocol produces hypoxic and ischemic neuronal and glial cell injury in the cortex, white matter, and putamen [9, 18-20]. Briefly, we lowered the inspired fraction of oxygen $\left(\mathrm{FiO}_{2}\right)$ to 0.10 for $45 \mathrm{~min}$, fol- lowed by $5 \mathrm{~min}$ of room air which is required for successful cardiac resuscitation. The endotracheal tube was then clamped for $7 \mathrm{~min}$ to produce asphyxia. We resuscitated the piglets with chest compressions and epinephrine $(0.1 \mathrm{mg} / \mathrm{kg}$ i.v.). Piglets that did not resuscitate by 3 min of compressions were excluded. Sham piglets received the same anesthesia and surgery but did not undergo hypoxia-ischemia.

\section{Temperature}

Some piglets remained normothermic at a goal rectal temperature of $37.5-39.5^{\circ} \mathrm{C}$ (normothermia for pigs) with warming blankets and a heating lamp (Fig. 1). Piglets randomized to receive hypothermia had whole-body cooling initiated $2 \mathrm{~h}$ after resuscitation from hypoxia-ischemia or the equivalent time in shams. Hypothermia was induced with a cooling blanket and ice-packs to achieve a goal rectal temperature of $32^{\circ} \mathrm{C}$. We delayed hypothermia induction by $2 \mathrm{~h}$ to mimic the cooling delays that may occur during clinical treatment of HIE. Some piglets received whole-body rewarming at $4{ }^{\circ} \mathrm{C} / \mathrm{h}$. Rewarming was accomplished by increasing the water temperature that circulated through the blanket and applying warm packs. Rewarming did not overshoot the normothermic goal temperature. Sham piglets received the same temperature regimen as the HI piglets.

\section{Cerebral Signal Acquisition}

We synchronously sampled the LDF, NIRS $\mathrm{rSO}_{2}$ and $\mathrm{HbT}$, ICP, and MAP at a sample rate of $100 \mathrm{~Hz}$ with ICM+ software (Cambridge University, Cambridge, UK). The HbT was obtained from NIRS measurements at an 805-nm wavelength, which is isosbestic 


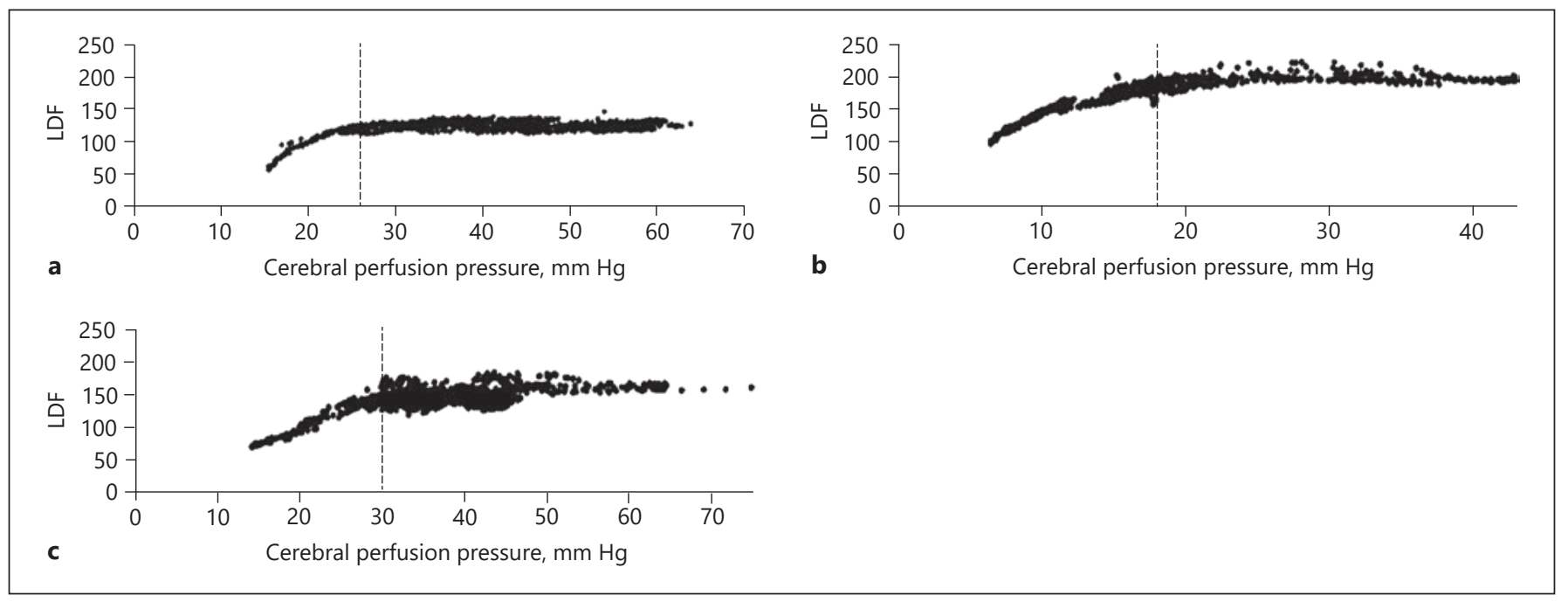

Fig. 2. Examples of cerebral blood flow measurements by laser Doppler flowmetry (LDF) in 3 piglets. The vertical line demarcates each piglet's lower limit of autoregulation. a, b Two different pig- lets that received hypoxic-ischemic (HI) brain injury and hypothermia. c HI-injured piglet with normothermia. to both deoxy- and oxyhemoglobin [6]. All signals were averaged in 10-s consecutive windows. This operation resamples the data at $0.1 \mathrm{~Hz}$ and eliminates high-frequency noise from respiration and pulse, but still permits signal analyses of oscillations $<0.05 \mathrm{~Hz}$ [15]. The CPP was calculated as MAP - ICP. Changes in CBF were estimated by $\mathrm{LDF}$ and $\mathrm{rSO}_{2}$ [9-11]. Fluctuations in $\mathrm{CBV}$ were estimated by ICP and HbT [6].

\section{Identifying the LLA}

After obtaining data for at least $2-3 \mathrm{~h}$ during normotension with an apparent LDF autoregulatory plateau, we slowly inflated the balloon catheter in the femoral vein over $2-3 \mathrm{~h}$, using a syringe pump to decrease venous return. In rewarmed pigs, the balloon catheter inflation began after normothermia was reached. A scatter plot was generated between CPP and LDF for each piglet across the entire 2-3 $\mathrm{h}$ period of balloon catheter inflation (Fig. 2). We then identified each piglet's LLA by using piecewise regression of the plot of LDF as a function of CPP (SigmaStat 3.1, Systat, San José, CA, USA). We reported the piglets' LLA values previously (online suppl. Table 1; for all online suppl. material, see www.karger.com/doi/10.1159/000499425) [911]. The investigator who calculated the LLAs (J.K.L.) was blinded to the time- and frequency-domain indices.

\section{Frequency- and Time-Domain Analytics}

We summarize the frequency domain analytics of autoregulation and their different interpretations. When blood pressure is on the autoregulatory plateau, the input (MAP or CPP) wave is not transmitted to the output $\mathrm{CBF}$. This generates low coherence and low gain between blood pressure and CBF. Functional vasoreactivity is identified when an increase in blood pressure causes a decrease in $\mathrm{CBV}$, thereby generating coherence with a negative phase shift (blood pressure and CBV are out of phase). When blood pressure is below the LLA with pressure-passive CBF and CBV, blood pressure is coherent to both $\mathrm{CBF}$ and $\mathrm{CBV}$ with a positive phase shift (blood pressure and CBF or CBV are in phase) and a high gain.
In the time domain, the blood pressure along the autoregulatory plateau generates a near-zero correlation between blood pressure and CBF. Functional vasoreactivity results in a negative correlation between blood pressure and $\mathrm{CBV}$ with a negative phase shift (out-of-phase). When blood pressure is below the LLA, there is a positive correlation between blood pressure and $\mathrm{CBV}$ or $\mathrm{CBF}$ with a positive phase shift (in-phase).

\section{Frequency- and Time-Domain Signal Processing}

We exported the data from ICM+ to MATLAB (Mathworks Inc., Natick, MA, USA), and an investigator blinded to the piglet treatment group (R.G.) calculated the autoregulation and vasoreactivity indices offline. Autoregulation indices were generated from 3 pairs of input and output signals: (1) MAP and $\mathrm{rSO}_{2},(2)$ $\mathrm{CPP}$ and LDF, and (3) MAP and LDF. We also calculated vasoreactivity indices from 3 pairs of input and output signals: (1) MAP and HbT, (2) CPP and HbT, and (3) MAP and ICP. Though only the MAP and NIRS $\mathrm{rSO}_{2}$ and $\mathrm{HbT}$ are routinely available during neonatal neurocritical care, for comparative purposes, we included the additional signal pairs commonly used in translational and adult autoregulation studies. The relationship between transcranial Doppler (analogous to laser Doppler flux) and either MAP [21] or CPP [22], depending on the availability of ICP monitoring to calculate CPP, has been well described as an autoregulation measure. Classic vasoreactivity monitoring in adult and pediatric studies has been derived from the pressure reactivity index, with MAP as the input and ICP as the output $[23,24]$.

Each piglet's signals were partitioned into consecutive 6-min epochs. This method provides a balance between having an adequate time span in each epoch that is long enough to accurately detect events that last $1 \mathrm{~min}$ [25], but short enough to obtain multiple measurements with perfusion pressure above or below the LLA. This ensured autoregulation index comparisons in equivalent time epochs. The 6-min epoch approximates the epoch that is frequently used in correlation analyses [26] and exceeds the epoch 
length of other coherence studies using short datasets in the lowfrequency band [27].

We used 4 mathematical algorithms to calculate indices from spectral coherence $(\mathrm{COH})$, cosine of the cross-spectral phase, gain, and Pearson's correlation coefficient for each pair of signals. These methods are described in detail below. In total, this method generated 12 autoregulation indices and 12 vasoreactivity indices.

A representative index value for dysfunctional autoregulation was generated for each piglet by averaging all values obtained from epochs with a CPP of $\geq 3 \mathrm{~mm} \mathrm{Hg}$ below the LLA. Similarly, a representative index value for functional autoregulation was calculated as the average of all values from epochs with a CPP of $\geq 3 \mathrm{~mm}$ $\mathrm{Hg}$ above the LLA. Thus, each piglet had 1 index for dysfunctional autoregulation (blood pressure below the LLA), and 1 for functional autoregulation (blood pressure above the LLA) from each signal pair (e.g., MAP and HbT) and mathematical algorithm (e.g., cosine of the cross-spectral phase). We excluded data from CPP of $<3 \mathrm{~mm} \mathrm{Hg}$ above or below the LLA to clearly distinguish dysfunctional from functional autoregulation.

\section{$\mathrm{COH}$ (Frequency Domain)}

In piglets, spontaneous fluctuations in MAP and ICP and the corresponding changes in $\mathrm{rSO}_{2}$ and $\mathrm{HbT}$ operate in the time scale of approximately $50-300 \mathrm{~s}[6,15]$. Coherence was calculated in the frequency range $0.003-0.02 \mathrm{~Hz}(50-333 \mathrm{~s})$ where piglet autoregulation is detectable. Several methods can be used to estimate coherence, including the multivariate autoregressive (MAR) approach, the Welch periodogram approach [28], and the smoothing of spectral quantities or tapering approach $[27,29]$. Because the MAR method is most suitable for datasets with a small number of samples within relatively short time epochs, [30] we used this approach. This is also consistent with methodology in other studies with short epochs $[27,31]$.

We modeled the data using an MAR process with model order determined by the Akaike information criterion [32]. The model order varied between 28 and 40 for different datasets. For consistency, we set a model order of 40 for all datasets. We estimated the regression coefficients through a modified Yule-Walker approach $[27,30]$ and calculated a covariance matrix from the residual errors. The MAR coefficients and error covariance matrix were used to calculate the cross-spectrum between the 2 signals and the auto (power) spectra of the 2 signals [30]. Then, we calculated the $\mathrm{COH}$ as the ratio of the square of the magnitude of the cross-spectrum to the product of the auto-spectra of the 2 signals. $\mathrm{COH}$ is a continuous measure that approaches 1 as two signals become increasingly associated but does not discern the presence of phase shift between two signals that have power in a common frequency. In cases of perfect association, the $\mathrm{COH}$ is 1 regardless of the phasic relationship. $\mathrm{COH}$ is zero when the 2 signals are not associated. We used the maximum $\mathrm{COH}$ within the frequency $0.003-0.02 \mathrm{~Hz}$ for the statistical analysis.

\section{Cosine of the Cross-Spectral Phase (Frequency Domain)}

We identified the frequency band with maximum $\mathrm{COH}$ within the frequency range $0.003-0.02 \mathrm{~Hz}$ where piglet autoregulation is detectable $[6,15]$. We then calculated phase shift between the input and output signals using the cross-spectrum at the frequency band with maximum $\mathrm{COH}$. The emphasis on studying phase shift in coherent data is similar to wavelet transform phase shift methodology that calculates phase only when coherence exceeds 0.48 [33]. However, our method differed, in that we did not use a coherence threshold. Rather, we calculated the phase in the frequency band of maximum $\mathrm{COH}$, and we did not require the data to pass a predetermined $\mathrm{COH}$ limit. The phase value was then cosine-transformed for analysis (phase).

\section{Gain (Frequency Domain)}

We calculated the input-output transfer gain as the ratio of the magnitude of the cross-spectrum to the power spectrum of the input signal [14]. The gain value at the frequency of maximum coherence in the range $0.003-0.02 \mathrm{~Hz}$ was used for the statistical analysis.

\section{Pearson's Correlation Coefficient (Time Domain)}

We calculated the Pearson's correlation coefficient (COR) between signal pairs averaged in 10 -s epochs. When autoregulation is functional, and MAP is above the LLA and along the autoregulatory plateau, the analysis of blood pressure (MAP or CPP) and $\mathrm{CBF}$ $\left(\mathrm{LDF}\right.$ or $\mathrm{rSO}_{2}$ ) yields a negative or near-zero COR (incoherent or coherent with a phase shift). When MAP is below the LLA during dysfunctional autoregulation, COR approaches 1 and becomes increasingly positive with progressive impairments in autoregulation (coherent with a minimal phase shift).

The inverse relationship between blood pressure (MAP or $\mathrm{CPP}$ ) and CBV (ICP or HbT) during functional vasoreactivity generates a negative COR that approaches -1 with robust vasoreactivity (coherent with a phase shift). Dysfunctional and pressure-passive vasoreactivity yields a positive COR that approaches 1 (coherent with minimal phase shift).

\section{Statistical Analysis}

We used receiver operating characteristics (ROC) analyses to describe each index's ability to distinguish functional autoregulation or vasoreactivity (CPP above the LLA) from dysfunctional autoregulation or vasoreactivity (CPP below the LLA). The $95 \%$ confidence interval (CI) of the area under the ROC curve (AUC) was determined by bootstrapping piglets with $>1,000$ replications for each index [34]. We estimated the index AUC and CI for all piglets and separately for the HI piglet group and the sham group. Based on visual inspection of a graph of AUCs (Fig. 3, 4), the indices with the highest estimated AUCs were placed into "group 1" and those with the lowest AUCs into "group 2." We also estimated AUCs and 95\% bootstrap CIs for piglets stratified by normothermia, sustained hypothermia, or rewarming.

Then, we used a pairwise bootstrap approach to compare each index's AUC against all others. We conducted these analyses using data from all piglets and separately among HI piglets and among shams. We used the bootstrap method to resample piglets while accounting for each piglet having multiple measures at different instances, and we compared the resulting distribution of estimated AUC for each index pair. We randomly permuted the index type of each piglet to simulate an environment under the null hypothesis that the indices are equally good measures, as is recommended for hypothesis testing with the bootstrap method [35]. We compared the difference in AUC between each index pair to the bootstrap-constructed distribution of differences under the null hypothesis of no difference (equivalent to a $\Delta$ [difference] in AUC of $0)$ [36]. Because the number of index pairs is large (24 indices, choose $2=276$ comparisons), we adjusted for multiple comparisons to maintain an overall type 1 error rate of 0.05 using Benjamini and Hochberg's method [37]. 
Fig. 3. Area under the curve (AUC) from the receiver operating characteristics analysis of each index's ability to discriminate CPP below the LLA from CPP above the LLA in all piglets $(n=66)$. The indices were divided into groups 1 and 2 based on visual inspection of the AUCs. The dashed line separates groups 1 and 2 .
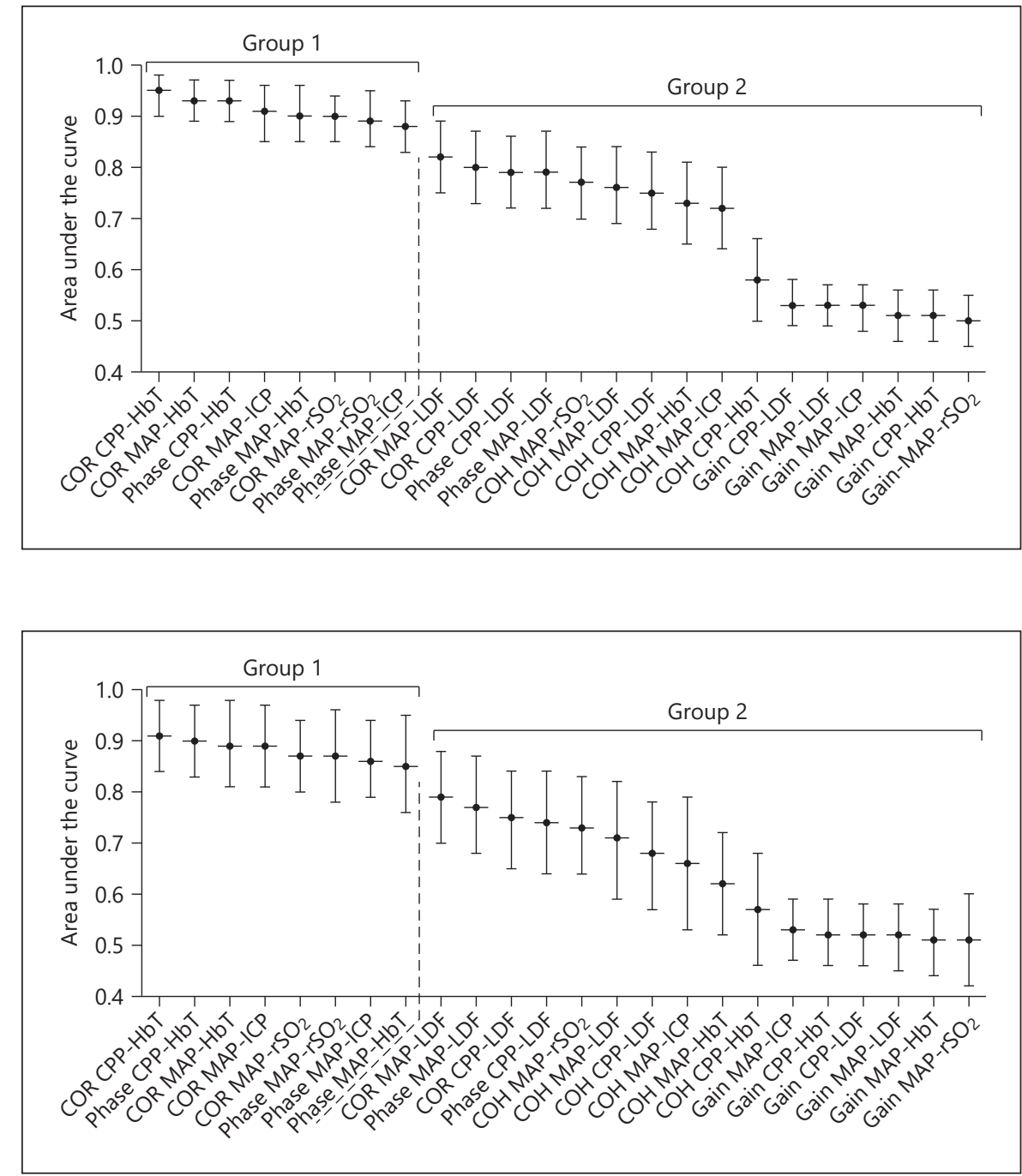

Fig. 4. Area under the curve (AUC) from the receiver operating characteristics analysis of each index's ability to discriminate CPP below the LLA from CPP above the LLA in 35 piglets with hypoxic-ischemic brain injury. The indices were divided into groups 1 and 2 based on visual inspection of the AUCs. The dashed line separates groups 1 and 2 . for the CPP below the LLA. The mean (standard deviation) number of 6-min epochs in periods with CPP below or CPP above the LLA was $46.6 \pm 44.9$ (range 1-249) and $131.7 \pm 94.8$ (range 12-395), respectively, for all piglets in all experimental conditions. The mean values of each index for CPP above or below the LLA are shown in online supplementary Table 2.

\section{Comparison of the Indices' Abilities to Discriminate between CPP above and CPP below the LLA in All Piglets}

The AUC values with 95\% CI for the COR, phase, $\mathrm{COH}$, and gain indices are shown in Figure 3. Indices in group 1 demonstrated a high discriminatory ability to distinguish whether CPP was above or below the LLA. The group 1 indices were $\mathrm{COR}_{\mathrm{CPP}-\mathrm{HbT}}, \mathrm{COR}_{\mathrm{MAP}-\mathrm{HbT}}$, Phase $_{\mathrm{CPP}-\mathrm{HbT}}$, COR $\mathrm{MAP}-\mathrm{ICP}$, Phase $_{\mathrm{MAP}-\mathrm{HbT}}, \mathrm{COR}_{\mathrm{MAP}-\mathrm{rSO} \text {, }}$ 
Table 1. Statistically significant pairwise comparisons of the receiver operating characteristics AUC for autoregulation and vasoreactivity indices in all 66 piglets

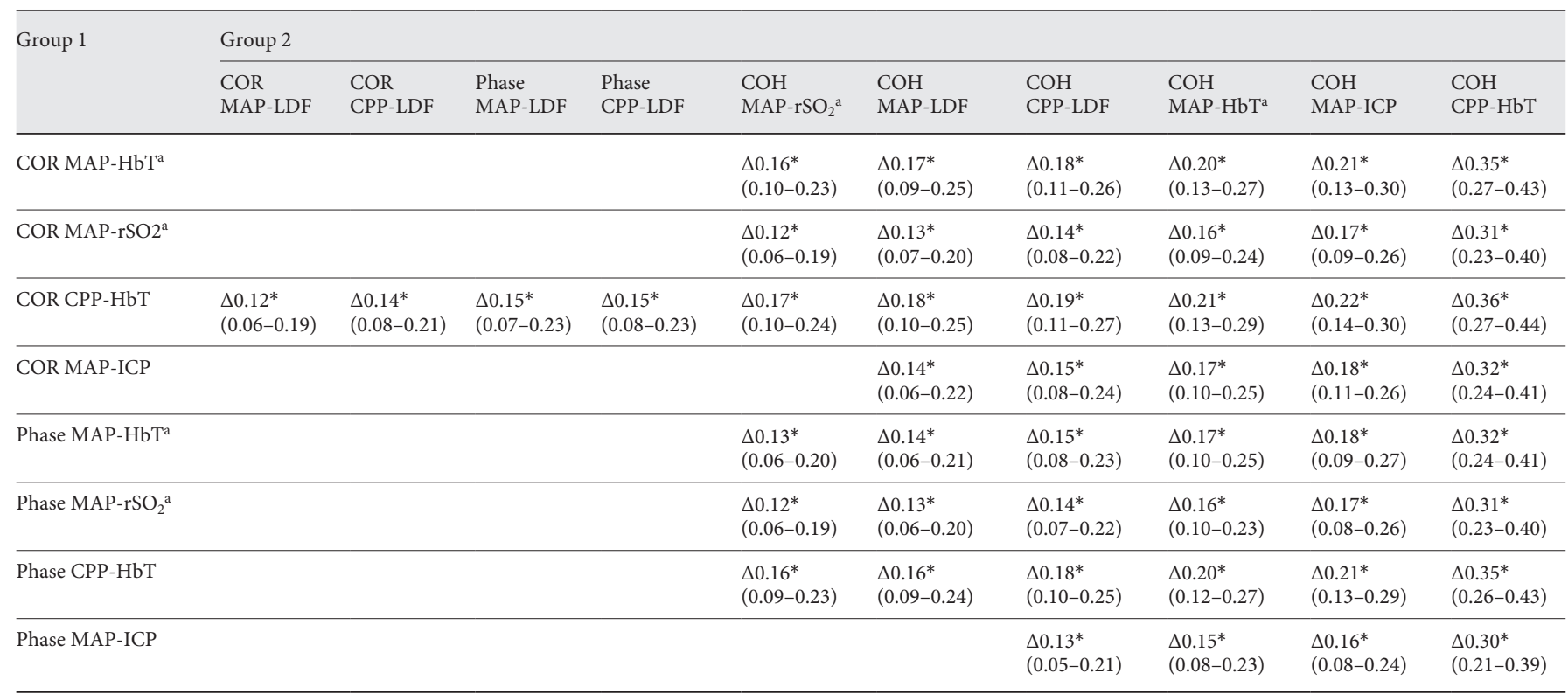

Data shown represent the difference in area under the curve (AUC), i.e., group 1 index - group 2 index $(\Delta)$ and $95 \%$ confidence intervals. * Adjusted $p<0.05$. COR, correlation MAP, mean arterial pressure; LDF, laser Doppler flowmetry; CPP, cerebral perfusion pressure; Phase, cosine of the cross-spectral phase; $\mathrm{COH}_{\text {, spectral coherence; } \mathrm{rSO}}$, near-infrared spectroscopy regional oxyhemoglobin saturation; HbT, near-infrared spectroscopy relative total tissue hemoglobin; ICP, intracranial pressure.

a The index was derived from parameters that are available during neonatal clinical care.

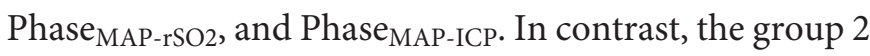
indices had a modest, and, in some cases, a poor discriminatory ability. The group 2 indices were $\mathrm{COR}_{\mathrm{MAP}-\mathrm{LDF}}$, COR $_{\text {CPP-LDF, }}$ Phase CCP-LDF, Phase MAP-LDF $_{\text {, }}, \mathrm{COH}_{\text {MAP-rSO2, }}$, $\mathrm{COH}_{\text {MAP-LDF }}, \mathrm{COH}_{\mathrm{CPP}-\mathrm{LDF}}, \mathrm{COH}_{\mathrm{MAP}-\mathrm{HbT}}, \mathrm{COH}_{\text {MAP-ICP}}$, $\mathrm{COH}_{\mathrm{CPP}-\mathrm{HbT}}, \quad \mathrm{Gain}_{\mathrm{CPP}-\mathrm{LDF}}, \quad \mathrm{Gain}_{\text {MAP-LDF}}$, Gain $_{\text {MAP- }}$ ICP, Gain ${ }_{\mathrm{MAP}-\mathrm{HbT}}$, Gain ${ }_{\mathrm{CPP}-\mathrm{HbT}}$, and Gain MAP-rSO2. .

Pairwise comparisons revealed no significant AUC differences among the indices of group 1, suggesting comparable discriminatory ability across these metrics (adjusted $p>0.05$ for all comparisons; Fig. 3 ). Several indices in group 1 had significantly higher AUCs than those in group 2 (Table 1). The AUCs of all group 1 indices significantly exceeded that of each gain metric (adjusted $p<$ 0.05 for all comparisons; data not shown).

\section{Comparison of the Indices after HI Brain Injury}

Among piglets with $\mathrm{HI}$ injury, no statistical differences in AUC were observed among the group 1 indices (Fig. 4). Several group 1 indices had a significantly higher AUC than several group 2 indices (Table 2). All gain metrics had a significantly lower AUC than each of group 1's indices (adjusted $p<0.05$ for all comparisons; data not shown).

Autoregulation and Neonatal Brain

Hypoxia-Ischemia
Comparison of the Indices after the Sham Procedure

Among the sham piglets, the AUC did not statistically differ between any group 1 indices. Several group 1 indices had a higher AUC than group 2 indices (Table 3). Every gain index had a lower AUC than each group 1 index (adjusted $p<0.05$ for all comparisons; data not shown).

\section{Impact of Hypoxia-Ischemia or Temperature}

on the Indices

The effects of HI brain injury and temperature on the indices' ability to distinguish CPP below or above the LLA are shown in Table 4. Hypoxia-ischemia and temperature did not significantly affect the ability to diagnose CPP below or above the LLA in any group 1 index $(p>0.05$ for all comparisons). Among the group 2 indices, hypoxia-ischemia decreased the accuracy of $\mathrm{COH}_{\mathrm{MAP}-\mathrm{LDF}}$ and $\mathrm{COH}_{\mathrm{M} \text { - }}$ AP-HbT to below that of the sham procedure $(p<0.05$ for each). $\mathrm{COH}_{\text {MAP-ICP }}$ performed significantly better during hypothermia than during normothermia $(p<0.05)$. The discriminatory ability of $\mathrm{COH}_{\mathrm{CPP}-\mathrm{LDF}}$ was greater in hypothermia than in rewarming $(p<0.05)$. COR $_{\text {MAP-LDF }}$ performed better in normothermia than in rewarming $(p<$ $0.05)$. Finally, Gain ${ }_{\mathrm{CPP}-\mathrm{LDF}}$ had greater diagnostic ability after rewarming than during normothermia $(p<0.05)$. 
Table 2. Statistically significant pairwise comparisons of the receiver operating characteristics AUC for autoregulation and vasoreactivity indices in 35 piglets with hypoxic-ischemic brain injury

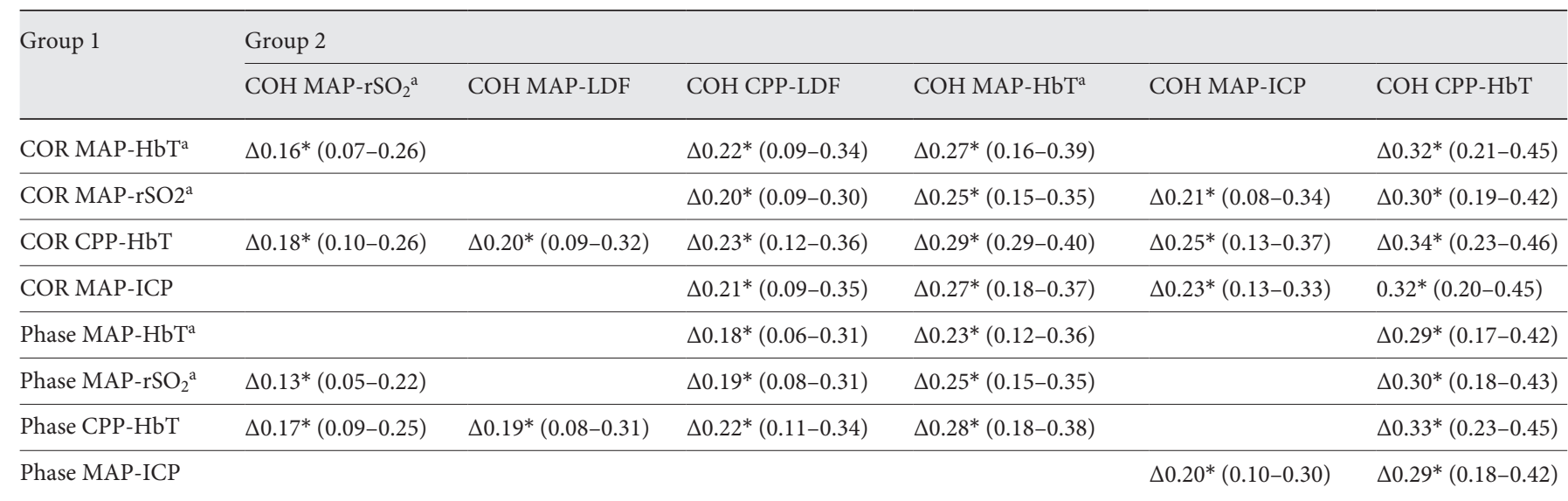

Data represent the difference in area under the curve (AUC), i.e., group 1 index - group 2 index $(\Delta)$ and $95 \%$ confidence intervals. ${ }^{*}$ Adjusted $p<0.05$. COH, spectral coherence; CPP, cerebral perfusion pressure; LDF, laser Doppler flowmetry; MAP, mean arterial pressure; HbT, near-infrared spectroscopy relative total tissue hemoglobin; COR, correlation; ICP, intracranial pressure; $\mathrm{rSO}_{2}$, near-infrared spectroscopy regional oxyhemoglobin saturation; Phase, cosine of the crossspectral phase.

a The index was derived from parameters that are available during neonatal clinical care.

Table 3. Statistically significant pairwise comparisons of the receiver operating characteristics AUC for autoregulation and vasoreactivity indices in 31 piglets that underwent the sham procedure

\begin{tabular}{lll}
\hline Group 1 & \multicolumn{2}{l}{ Group 2 } \\
\cline { 2 - 3 } & COH MAP-ICP & COH CPP-HbT \\
\hline COR MAP-HbT $^{\mathrm{a}}$ & & $\Delta 0.36^{*}(0.26-0.47)$ \\
COR MAP-rSO2 $^{\mathrm{a}}$ & & $\Delta 0.32^{*}(0.22-0.43)$ \\
COR CPP-HbT $^{*}$ & $\Delta 0.17^{*}(0.08-0.28)$ & $\Delta 0.37^{*}(0.26-0.48)$ \\
COR MAP-ICP $^{\mathrm{N}}$ & & $\Delta 0.32^{*}(0.20-0.43)$ \\
Phase MAP-HbT $^{\mathrm{a}}$ & & $\Delta 0.34^{*}(0.23-0.45)$ \\
Phase MAP-rSO $^{\mathrm{a}}$ & & $\Delta 0.33^{*}(0.22-0.45)$ \\
Phase CPP-HbT & & $\Delta 0.36^{*}(0.26-0.47)$ \\
Phase MAP-ICP & & $\Delta 0.30^{*}(0.18-0.42)$ \\
\hline
\end{tabular}

Data represent the difference in area under the curve (AUC), i.e., group 1 index - group 2 index $(\Delta)$ and $95 \%$ confidence interval. * Adjusted $p<0.05$. COH, spectral coherence; CPP, cerebral perfusion pressure; LDF, laser Doppler flowmetry; MAP, mean arterial pressure; $\mathrm{HbT}$, near-infrared spectroscopy relative total tissue hemoglobin; COR, correlation; ICP, intracranial pressure; $\mathrm{rSO}_{2}$, near-infrared spectroscopy regional oxyhemoglobin saturation; phase, cosine of the cross-spectral phase.

a The index was derived from parameters that are available during neonatal clinical care.

\section{Discussion}

We compared the diagnostic ability of frequency-domain ( $\mathrm{COH}$, phase, and gain) and time-domain (COR) autoregulation and vasoreactivity indices to discriminate $\mathrm{CPP}$ below the LLA from CPP above the LLA in a neonatal piglet model of HIE, hypothermia, rewarming, and controlled hypotension. Both COR and phase had a high accuracy for identifying pressure passivity, whereas $\mathrm{COH}$ alone and gain at the bandwidth of maximal coherence performed poorly. Because both COR and phase quantify the phase shift between the input (MAP or CPP) and output $\left(\mathrm{rSO}_{2}, \mathrm{LDF}\right.$, $\mathrm{HbT}$, or ICP) signals involved in autoregulation, our findings indicate that phase shift is the most important component of the relationship between blood pressure and CBF or CBV to capture when monitoring autoregulation and vasoreactivity in the HI-injured, developing brain.

Coherence measures the transmission of blood pressure waves to the $\mathrm{CBF}$ and $\mathrm{CBV}$, and gain measures the magnitude of this transmission. The relatively low discriminatory power of $\mathrm{COH}$ alone and gain suggest that perfusion pressure waves are similarly transmitted to the CBF and CBV regardless of whether blood pressure is above or below the LLA. $\mathrm{COH}$ and gain do not take phase shift into account, and autoregulatory vasoreactivity is multiphasic. When blood pressure is on the autoregulatory plateau, blood pressure and CBV are out of phase. When blood pressure decreases to below the LLA, blood pressure and CBV are pres- 
Table 4. Statistically significant, pairwise comparisons of the receiver operating characteristics AUC between $35 \mathrm{HI}$ and 31 sham piglets, and between normothermia $(n=35)$, sustained hypothermia $(n=20)$, and rewarming $(n=11)$

\begin{tabular}{|c|c|c|c|c|c|}
\hline Experimental condition & Metric & $\triangle \mathrm{AUC}$ & Lower limit 95\% CI & Upper limit 95\% CI & $p$ value \\
\hline HI vs. sham piglets & $\begin{array}{l}\text { COH CPP-LDF } \\
\text { COH MAP-HbT }^{a}\end{array}$ & $\begin{array}{l}-0.16^{\mathrm{b}} \\
-0.25^{\mathrm{b}}\end{array}$ & $\begin{array}{l}-0.3 \\
-0.40\end{array}$ & $\begin{array}{l}-0.02 \\
-0.10\end{array}$ & $\begin{array}{l}0.037 \\
0.005\end{array}$ \\
\hline Hypothermia vs. normothermia & COH MAP-ICP & $0.22^{\mathrm{c}}$ & 0.06 & 0.38 & 0.019 \\
\hline Hypothermia vs. rewarming & COH CPP-LDF & $0.21^{\mathrm{d}}$ & 0.03 & 0.39 & 0.046 \\
\hline Normothermia vs. rewarming & Gain CPP-LDF & $-0.20^{f}$ & -0.35 & 0.0 & 0.009 \\
\hline
\end{tabular}

$\triangle \mathrm{AUC}$, difference in area under the curve; $\mathrm{CI}$, confidence interval; $\mathrm{HI}$, hypoxic-ischemic; $\mathrm{COH}$, spectral coherence; $\mathrm{CPP}$, cerebral perfusion pressure; LDF, laser Doppler flowmetry; MAP, mean arterial pressure; HbT, near-infrared spectroscopy relative total tissue hemoglobin; ICP, intracranial pressure; COR, correlation.

a The index was derived from parameters that are available during neonatal clinical care.

$\mathrm{b}$ The index had lower diagnostic ability after hypoxia-ischemia than after the sham procedure.

${ }^{c}$ The index had greater diagnostic ability during sustained hypothermia than during normothermia.

$\mathrm{d}$ The index had greater diagnostic ability during sustained hypothermia than after rewarming.

e The index had greater diagnostic ability during normothermia than after rewarming.

$\mathrm{f}$ The index had greater diagnostic ability after rewarming than during normothermia.

sure-passive and in phase. We theorize that $\mathrm{COH}$ alone and gain poorly distinguished pressure reactivity from pressure passivity over the examined frequency band because $\mathrm{COH}$ and gain do not assess the phase relationship.

Few neonatal studies have directly compared frequency- and time-domain methods within the same subject. One study of 12 piglets reported that $\mathrm{COH}$ alone poorly characterized autoregulation [38]. Here, in a larger piglet cohort, we verified that $\mathrm{COH}$ alone is indeed a poor autoregulation metric, including after $\mathrm{HI}$ injury. In a clinical study of 60 premature neonates that compared NIRS frequency-and time-domain methods, the authors concluded that the time domain is a more accurate and easier method for neonatal autoregulation monitoring [39]. However, they did not evaluate phase in the frequency bandwidth of maximum $\mathrm{COH}$. Moreover, their study along with most clinical neonatal studies did not have a comparative and continuous $\mathrm{CBF}$ measure to define the state of autoregulation. The advantage of testing mathematical algorithms in a piglet model is that LDF can be used continuously to detect $\mathrm{CBF}$, accurately determine when blood pressure is above or below the LLA, and then generate a gold standard against which different algorithms can be tested within the same animal. With these methods, we were able to perform systematic comparative analyses among autoregulation metrics and identify phase and COR approaches as those that should move forward to clinical validation studies.

Autoregulation and Neonatal Brain

Hypoxia-Ischemia
Phase and COR did not show a statistical difference when calculated from the NIRS $\mathrm{HbT}$ or $\mathrm{rSO}_{2}$. Multiple factors can influence $\mathrm{rSO}_{2}$, including fluctuations in the cerebral metabolic rate that occur with any changes in sedation or pain management, oxygen delivery, and temperature. Because HbT is calculated at the $805-\mathrm{nm}$ wavelength that is isosbestic to both oxygenated and deoxygenated hemoglobin, it should be relatively unaffected by changes in cerebral metabolic rate or oxygen delivery provided that the total hemoglobin, carbon dioxide, and sedation levels remain constant. We maintained normocapnea within a constant plane of anesthesia in our piglets, and we did not use hemorrhage as a method to induce hypotension. Accordingly, the indices derived from $\mathrm{rSO}_{2}$ performed as well as those from $\mathrm{HbT}$. However, treatment and physiologic conditions cannot be as rigorously controlled in clinical practice. Whether the accuracy of autoregulation and vasoreactivity indices from $\mathrm{rSO}_{2}$ and $\mathrm{HbT}$ differ during clinical treatment of HIE deserves further study.

Monitoring methods should ideally be validated in preclinical models and controlled physiologic experiments before their deployment clinically. Among the indices calculated from parameters available during routine neonatal clinical care (specifically MAP, $\mathrm{HbT}$, and $\mathrm{rSO}_{2}$ ), the $\mathrm{HI}$ injury significantly decreased the ability of $\mathrm{COH}_{\mathrm{MAP}-\mathrm{HbT}}$ to detect the pressure passivity relative to that of the sham procedure. The fact that HI injury did not affect the accu- 
racy of phase and COR supports the use of these metrics in clinical HIE studies. Moreover, temperature did not affect the accuracy of indices derived from MAP and HbT or $\mathrm{rSO}_{2}$, which supports their use in clinical studies.

We did not induce hypotension during rewarming because we assumed that the change in cerebral metabolic rate and $\mathrm{CBF}$ coupling would confound our measurements. $\mathrm{COR}_{\mathrm{MAP}-\mathrm{LDF}}$ had lower diagnostic accuracy after rewarming than during normothermia, whereas Gain ${ }_{\mathrm{CPP}}$ LDF performed better after rewarming than during normothermia. Further testing on autoregulation monitoring during and after rewarming is critical if these metrics are to be considered for clinical use. The closest clinical correlate to LDF is transcranial Doppler. Autoregulatory vasoreactivity remains disrupted after the completion of rewarming from clinical hypothermic cardiopulmonary bypass [40], hypothermic treatment for adult traumatic brain injury [41], and in rats [42]. In neonatal HIE, potential oxidative stress [16] and inflammation [17] during rewarming could affect the magnitude and change in subsequent vasoreactive responses as the blood pressure changes. This could affect whether an index can detect the relative change in vascular reactivity when blood pressure is above versus below the LLA. Moreover, the piglet rewarming rate of $4^{\circ} \mathrm{C} / \mathrm{h}$ in this study is faster than typical clinical rewarming rates for HIE, which are usually $0.25-0.5^{\circ} \mathrm{C} / \mathrm{h}$. Studies using more clinically relevant rewarming rates are needed.

We have determined that phase and COR have the best ability to discriminate pressure passivity versus pressure reactivity in piglets. We could not examine neuropathologic injury or behavioral outcomes in our piglets because of the severe injury that occurs from induced hypotension. Nonetheless, phase and COR using MAP and NIRS $\mathrm{HbT}$ or $\mathrm{rSO}_{2}$ appear to be reliable candidate measures for clinical autoregulation studies in HIE.

We specifically analyzed maximal $\mathrm{COH}$. Though it is possible that our $\mathrm{COH}$ estimates were not reliable, the phase calculations in the frequency band of maximal $\mathrm{COH}$ distinguished the blood pressure above and below the LLA with a high accuracy. Phase between 2 signals in a frequency band is only reliable if the $\mathrm{COH}$ meets the significance criterion at that frequency band. Thus, the excellent performance of phase suggests that our $\mathrm{COH}$ estimations were accurate. Additional studies are needed to examine relevant coherence thresholds in HIE.

Each piglet's LLA was identified using piecewise regression of a static LDF-CPP curve from the entire $2-3 \mathrm{~h}$ period of induced hypotension without a time series analysis. This method provides a gold standard LLA, and the LLAs were calculated by an investigator blinded to the au- toregulation indices. However, using a regression method to identify the LLA has similarities with time-domain COR, and this could potentially bias the index performance. Nevertheless, metrics from both the frequency and time domains accurately distinguished the blood pressure above and below the LLA. The top performing indices COR and phase in the bandwidth of maximal coherence showed no statistical AUC differences. Thus, our method of calculating the LLA did not favor the time domain over the frequency domain. Though we did not use alternative methods to calculate the LLA to double-check our findings, we visually verified the LLA by inspecting each piglet's LDF-CPP plot (examples in Fig. 2). Clinically, the goal of autoregulation monitoring is to detect the risk of neurologic injury before injury occurs, by identifying the LLA and maintaining the blood pressure above the LLA. We will explore whether phase or COR predict brain injury in clinical HIE in future studies.

The appropriate frequency bandwidth for detecting autoregulation and vasoreactivity signals has been the subject of much debate and varies widely $[1,5,43]$. Because we previously established that spontaneous fluctuations in CPP typically occur on the time scale of 50-300 s $[6,15]$, we focused our analyses within the frequency range $0.003-0.02 \mathrm{~Hz}$ (50-333 s). A recent study with constant frequency oscillations in CPP in piglets showed that the maximal phase shift between MAP and ICP (which reflects CBV fluctuations) occurs at $0.017 \mathrm{~Hz}(1 \mathrm{~min})$ [25]. This is within the range tested in our study.

The piglet methodology must be distinguished from clinical practice. The piglets were under anesthesia (lowdose isoflurane) to ensure comfort. Though higher doses of isoflurane may uncouple CBF [44], the low dose of isoflurane in our experiment still revealed apparent blood pressure ranges with an autoregulatory plateau, LLA, and pressure passivity [9-11] (Fig. 2). Clinically, babies receive a lighter level of sedation, which may cause changes in cerebral metabolic rate and movement. This can change the oxyhemoglobin concentration, which would be expected to have a greater effect on $\mathrm{rSO}_{2}$ than on $\mathrm{HbT}$ and introduce monitoring artifacts. Clinicians do not permit neonates to have extremely low blood pressure as we did with the piglets. We also separated the blood pressure ranges for above and below the LLA by $3 \mathrm{~mm} \mathrm{Hg}$ to clearly delineate functional from dysfunctional autoregulation. Differences in clinical autoregulatory function may be harder to detect with narrower blood pressure ranges.

We acknowledge several limitations. Only a few other studies have directly compared different autoregulation metrics in the piglet model. Without the necessary input 
from previous studies, such as the expected difference in the predictive power of the autoregulation indices and their respective measurement error, we were unable to quantify the power in this study to detect statistical differences. We only tested males, and data were analyzed retrospectively from 3 separate studies. We did not measure hypertension and autoregulatory function because newborn piglets frequently suffer cardiac failure before the upper limit of autoregulation can be reached. Though we calculated gain at the frequency of maximum $\mathrm{COH}$, we did not assess the statistical significance of the $\mathrm{COH}$ or use a $\mathrm{COH}$ threshold. Determining the statistical significance of $\mathrm{COH}$ prior to calculating gain may improve accuracy. Because the $\mathrm{COH}$ threshold for brain injury in HIE is not known, we used the maximal $\mathrm{COH}$ in this exploratory, preclinical study. We did not examine wavelet $\mathrm{COH}$ analysis [5] or neurovascular coupling [45].

\section{Conclusion}

In a neonatal piglet model of HI brain injury, phase and COR calculations between MAP and NIRS HbT or $\mathrm{rSO}_{2}$ accurately distinguished whether CPP was above or below the LLA. HI injury did not reduce the accuracy of these indices. In contrast, $\mathrm{COH}$ alone and gain were poor indicators of autoregulation and vasoreactivity. Candidate indices for clinical HIE autoregulation studies include phase and COR between MAP and $\mathrm{HbT}$ or $\mathrm{rSO}_{2}$.

\section{Acknowledgments}

We thank Claire Levine for her editorial assistance.

\section{Statement of Ethics}

Animal experiments conformed to internationally accepted standards and were approved by the Johns Hopkins University Animal Care and Use Committee.

\section{Disclosure Statement}

Some methods used to measure and monitor autoregulation as described in this manuscript were patented by The Johns Hopkins University, listing K.B. as a coinventor. These patents are exclusively licensed to Medtronic Inc. and K.B. received a portion of the licensing fee. J.K.L. received research support from Medtronic for a separate study. J.K.L. was also a paid Advisory Board member for Medtronic. This arrangement was reviewed and approved by the Johns Hopkins University in accordance with its conflict of inter-

est policies. Medtronic had no role in the study design, data collection and analysis, interpretation of results, writing, or decision to submit our manuscript for publication.

\section{Funding}

This work was supported by the National Institutes of Health, Bethesda, MD, USA (grant Nos. K08NS080984 and R01NS107417 to J.K.L., and R01NS060703 to RCK), an American Heart Association grant-in-aid (to A.N.M., R.B.G., and J.K.L.), and an American Heart Association Transformational Project Award (to J.K.L.).

\section{Author Contributions}

Dr. Govindan made substantial contributions to the conception and design of the study, data analysis, and interpretation of the data. He was involved in writing and revising the manuscript, and approved the final draft. Dr. Brady made substantial contributions to the conception and design of the study, data acquisition, data analysis, and interpretation of the data. He was involved in writing and revising the manuscript, and approved the final draft. Dr. Massaro made substantial contributions to the conception and design of the study, data analysis, and interpretation of the data. She was involved in writing and revising the manuscript, and approved the final draft. Dr. Perin made substantial contributions to the design of the study, data analysis, and interpretation of the data. She was involved in writing and revising the manuscript, and approved the final draft. Dr. Jennings made substantial contributions to the design of the study, data analysis, and interpretation of the data. She was involved in writing and revising the manuscript, and approved the final draft. Dr. du Plessis made substantial contributions to the conception and design of the study, data analysis, and interpretation of the data. He was involved in writing and revising the manuscript, and approved the final draft. Dr. Koehler made substantial contributions to the conception and design of the study, data acquisition, data analysis, and interpretation of the data. He was involved in writing and revising the manuscript, and approved the final draft. Dr. Lee made substantial contributions to the conception and design of the study, data acquisition, data analysis, and interpretation of the data. She was involved in writing and revising the manuscript, and approved the final draft.

References

Dev Neurosci 2018;40:547-559

DOI: $10.1159 / 000499425$
1 Massaro AN, Govindan RB, Vezina G, Chang $\mathrm{T}$, Andescavage NN, Wang Y, et al. Impaired cerebral autoregulation and brain injury in newborns with hypoxic-ischemic encephalopathy treated with hypothermia. J Neurophysiol. 2015 Aug;114(2):818-24.

2 Tekes A, Poretti A, Scheurkogel MM, Huisman TA, Howlett JA, Alqahtani E, et al. Apparent diffusion coefficient scalars correlate with near-infrared spectroscopy markers of cerebrovascular autoregulation in neonates cooled for perinatal hypoxic-ischemic injury. AJNR Am J Neuroradiol. 2015 Jan;36(1):18893. 
3 Burton VJ, Gerner G, Cristofalo E, et al. A pilot cohort study of cerebral autoregulation and 2-year neurodevelopmental outcomes in neonates with hypoxic-ischemic encephalopathy who received therapeutic hypothermia. BMC Neurol. 2015 Oct;15:209.

4 Lee JK, Poretti A, Perin J, Huisman TA, Parkinson C, Chavez-Valdez R, et al. Optimizing cerebral autoregulation may decrease neonatal regional hypoxic-ischemic brain injury. Dev Neurosci. 2017;39(1-4):248-56.

5 Tian F, Tarumi T, Liu H, Zhang R, Chalak L. Wavelet coherence analysis of dynamic cerebral autoregulation in neonatal hypoxic-ischemic encephalopathy. Neuroimage Clin. 2016 Jan;11:124-32.

6 Lee JK, Kibler KK, Benni PB, Easley RB, Czosnyka M, Smielewski P, et al. Cerebrovascular reactivity measured by near-infrared spectroscopy. Stroke. 2009 May;40(5):1820-6.

7 Edwards AD, Wyatt JS, Richardson C, Delpy DT, Cope M, Reynolds EO. Cotside measurement of cerebral blood flow in ill newborn infants by near infrared spectroscopy. Lancet. 1988 Oct;2(8614):770-1.

8 Soul JS, du Plessis AJ. New technologies in pediatric neurology. Near-infrared spectroscopy. Semin Pediatr Neurol. 1999 Jun;6(2):10110.

9 Lee JK, Yang ZJ, Wang B, Larson AC, Jamrogowicz JL, Kulikowicz E, et al. Noninvasive autoregulation monitoring in a swine model of pediatric cardiac arrest. Anesth Analg. 2012 Apr;114(4):825-36.

10 Larson AC, Jamrogowicz JL, Kulikowicz E, Wang B, Yang ZJ, Shaffner DH, et al. Cerebrovascular autoregulation after rewarming from hypothermia in a neonatal swine model of asphyxic brain injury. J Appl Physiol (1985). 2013 Nov;115(10):1433-42.

11 Lee JK, Brady KM, Mytar JO, Kibler KK, Carter EL, Hirsch KG, et al. Cerebral blood flow and cerebrovascular autoregulation in a swine model of pediatric cardiac arrest and hypothermia. Crit Care Med. 2011 Oct;39(10): 2337-45.

12 Tsuji M, duPlessis A, Taylor G, Crocker R, Volpe JJ. Near infrared spectroscopy detects cerebral ischemia during hypotension in piglets. Pediatr Res. 1998 Oct;44(4):591-5.

13 Soul JS, Hammer PE, Tsuji M, Saul JP, Bassan $\mathrm{H}$, Limperopoulos $\mathrm{C}$, et al. Fluctuating pressure-passivity is common in the cerebral circulation of sick premature infants. Pediatr Res. 2007 Apr;61(4):467-73.

14 O'Leary H, Gregas MC, Limperopoulos C, Zaretskaya I, Bassan H, Soul JS, et al. Elevated cerebral pressure passivity is associated with prematurity-related intracranial hemorrhage. Pediatrics. 2009 Jul;124(1):302-9.

15 Brady KM, Lee JK, Kibler KK, Smielewski P, Czosnyka M, Easley RB, et al. Continuous time-domain analysis of cerebrovascular autoregulation using near-infrared spectroscopy. Stroke. 2007 Oct;38(10):2818-25.

16 Santos PT, O'Brien CE, Chen MW, et al. Proteasome biology is compromised in white matter after asphyxic cardiac arrest in neonatal piglets. J Am Heart Assoc. 2018 Oct 16; 7(20):e009415.

17 Bisschops LL, Hoedemaekers CW, Mollnes TE, van der Hoeven JG. Rewarming after hypothermia after cardiac arrest shifts the inflammatory balance. Crit Care Med. 2012 Apr;40(4):1136-42.

18 Wang B, Armstrong JS, Reyes M, Kulikowicz E, Lee JH, Spicer D, et al. White matter apoptosis is increased by delayed hypothermia and rewarming in a neonatal piglet model of hypoxic ischemic encephalopathy. Neuroscience. 2016 Mar;316:296-310.

19 O’Brien CE, Santos PT, Kulikowicz E, et al. Hypoxia-ischemia and hypothermia independently and interactively affect neuronal pathology in neonatal piglets with shortterm recovery. Dev Neurosci. Forthcomin 2019.

20 Easley RB, Jennings J, Kibler K, Brady KM, Serratos B, Andropoulos DB, et al. Cerebrovascular autoregulation impairment is associated with elevations in plasma glial fibrillary acidic protein during congenital heart surgery (Abstracts from the American Heart Association's Emerging Science Series, April 25, 2012). Circulation. 2012;125: 2445-7.

21 Soehle M, Chatfield DA, Czosnyka M, Kirkpatrick PJ. Predictive value of initial clinical status, intracranial pressure and transcranial Doppler pulsatility after subarachnoid haemorrhage. Acta Neurochir (Wien). 2007 Jun; 149(6):575-83.

22 Zeiler FA, Cardim D, Donnelly J, Menon DK, Czosnyka M, Smielewski P. Transcranial doppler systolic flow index and ICP-derived cerebrovascular reactivity indices in traumatic brain injury. J Neurotrauma. 2018 Jan;35(2): $314-22$.

23 Aries MJ, Czosnyka M, Budohoski KP, Kolias AG, Radolovich DK, Lavinio A, et al. Continuous monitoring of cerebrovascular reactivity using pulse waveform of intracranial pressure. Neurocrit Care. 2012 Aug; 17(1): $67-76$.

24 Brady KM, Shaffner DH, Lee JK, Easley RB, Smielewski P, Czosnyka M, et al. Continuous monitoring of cerebrovascular pressure reactivity after traumatic brain injury in children. Pediatrics. 2009 Dec;124(6):e120512.

25 Fraser CD 3rd, Brady KM, Rhee CJ, Easley RB, Kibler K, Smielewski P, et al. The frequency response of cerebral autoregulation. J Appl Physiol (1985). 2013 Jul;115(1):52-6.

26 Brady K, Joshi B, Zweifel C, Smielewski P, Czosnyka M, Easley RB, et al. Real-time continuous monitoring of cerebral blood flow autoregulation using near-infrared spectroscopy in patients undergoing cardiopulmonary bypass. Stroke. 2010 Sep;41(9): 1951-6.

27 Barbieri R, Triedman JK, Saul JP. Heart rate control and mechanical cardiopulmonary coupling to assess central volume: a systems analysis. Am J Physiol Regul Integr Comp Physiol. 2002 Nov;283(5):R1210-20.

28 Halliday DM, Rosenberg JR, Amjad AM, Breeze P, Conway BA, Farmer SF. A framework for the analysis of mixed time series/ point process data-theory and application to the study of physiological tremor, single motor unit discharges and electromyograms. Prog Biophys Mol Biol. 1995;64(2-3):23778.

29 Timmer J, Lauk M, Pfleger W, Deuschl G. Cross-spectral analysis of physiological tremor and muscle activity. I. Theory and application to unsynchronized electromyogram. Biol Cybern. 1998 May;78(5):349-57.

30 Kay SM. Modern Spectral Estimation: Theory and Application. New Jersey: Prentice Hall; 1998.

31 Wyller VB, Barbieri R, Saul JP. Blood pressure variability and closed-loop baroreflex assessment in adolescent chronic fatigue syndrome during supine rest and orthostatic stress. Eur J Appl Physiol. 2011 Mar;111(3): 497-507.

32 Akaike H. A new look at the statistical model identification. IEEE Trans Automat Contr. 1974;19(6):716-23.

33 Liu X, Czosnyka M, Donnelly J, Cardim D, Cabeleira M, Hutchinson PJ, et al. Wavelet pressure reactivity index: a validation study. J Physiol. 2018 Jul;596(14):2797-809.

34 DiCiccio TJ, Efron B, Hall P, Martin MA, Canty AJ, Davison AC, et al. Bootstrap confidence intervals. Stat Sci. 1996;11(3):189-228.

35 Hall P, Wilson SR. Two guidelines for bootstrap hypothesis testing. Biometrics. 1991; 47(2):757-62.

36 Mandrekar JN. Receiver operating characteristic curve in diagnostic test assessment. J Thorac Oncol. 2010 Sep;5(9):1315-6.

37 Benjamini Y, Hochberg Y. Controlling the false discovery rate: A practical and powerful approach to multiple testing. J R Stat Soc B. 1995;57(1):289-300.

38 Hahn GH, Heiring C, Pryds O, Greisen G. Applicability of near-infrared spectroscopy to measure cerebral autoregulation noninvasively in neonates: a validation study in piglets. Pediatr Res. 2011 Aug; 70(2):166-70.

39 Eriksen VR, Hahn GH, Greisen G. Cerebral autoregulation in the preterm newborn using near-infrared spectroscopy: a comparison of time-domain and frequency-domain analyses. J Biomed Opt. 2015 Mar;20(3): 037009.

40 Joshi B, Brady K, Lee J, Easley B, Panigrahi R, Smielewski $\mathrm{P}$, et al. Impaired autoregulation of cerebral blood flow during rewarming from hypothermic cardiopulmonary bypass and its potential association with stroke. Anesth Analg. 2010 Feb;110(2):321-8.

41 Lavinio A, Timofeev I, Nortje J, Outtrim J, Smielewski P, Gupta A, et al. Cerebrovascular reactivity during hypothermia and rewarming. Br J Anaesth. 2007 Aug;99(2):23744. 
42 Ueda Y, Suehiro E, Wei EP, Kontos HA, Povlishock JT. Uncomplicated rapid posthypothermic rewarming alters cerebrovascular responsiveness. Stroke. 2004 Feb;35(2):6016.

43 Howlett JA, Northington FJ, Gilmore MM, Tekes A, Huisman TA, Parkinson C, et al.
Cerebrovascular autoregulation and neurologic injury in neonatal hypoxic-ischemic encephalopathy. Pediatr Res. 2013 Nov;74(5): 525-35.

44 Aksenov D, Eassa JE, Lakhoo J, Wyrwicz A, Linsenmeier RA. Effect of isoflurane on brain tissue oxygen tension and cerebral autoregu- lation in rabbits. Neurosci Lett. 2012 Aug; 524(2):116-8.

45 Chalak LF, Zhang R. New wavelet neurovascular bundle for bedside evaluation of cerebral autoregulation and neurovascular coupling in newborns with hypoxic-ischemic encephalopathy. Dev Neurosci. 2017;39(1-4):89-96. 